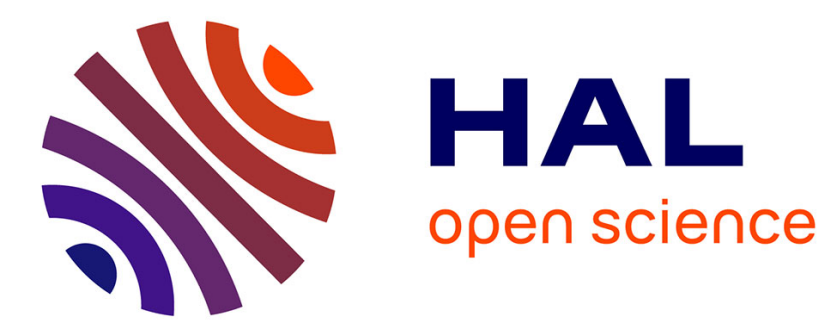

\title{
The Adaptive Sex in Stressful Environments
}

Benjamin Geffroy, Mathieu Douhard

\section{To cite this version:}

Benjamin Geffroy, Mathieu Douhard. The Adaptive Sex in Stressful Environments. Trends in Ecology and Evolution, 2019, 34 (7), pp.628-640. 10.1016/j.tree.2019.02.012 . hal-02434170

\section{HAL Id: hal-02434170 \\ https://hal.science/hal-02434170}

Submitted on 25 Oct 2021

HAL is a multi-disciplinary open access archive for the deposit and dissemination of scientific research documents, whether they are published or not. The documents may come from teaching and research institutions in France or abroad, or from public or private research centers.
L'archive ouverte pluridisciplinaire HAL, est destinée au dépôt et à la diffusion de documents scientifiques de niveau recherche, publiés ou non, émanant des établissements d'enseignement et de recherche français ou étrangers, des laboratoires publics ou privés.

\section{(ㄷ)(1) $\$$}

Distributed under a Creative Commons Attribution - NonCommerciall 4.0 International 


\section{The adaptive sex in stressful environments}

2

3

$4 \quad$ Benjamin Geffroy ${ }^{1 *_{+}}$and Mathieu Douhard ${ }^{2 *}$

5

6

7

8

9

10

11

12

13

14

15

16

17

18

19

20

22

23

24 trends.

*Both authors contributed equally to the manuscript

+Corresponding author: bgeffroy@ifremer.fr

Keywords

\section{Abstract}

21 While exposure to glucocorticoids around the time of sex determination in fish consistently

${ }^{1}$ MARBEC, Ifremer, Univ Montpellier, CNRS, IRD, Palavas-Les-Flots, France.

${ }^{2}$ Laboratoire d'Ecologie Alpine, CNRS UMR5553, Université de Savoie, Le Bourget du Lac, France.

Sex determination; Sex ratio; Maternal effect; Adaptive response; Glucocorticoids

The impact of early stress on juvenile development has intrigued scientists for decades, but the adaptive significance of such effects remains an ongoing debate. This debate has largely ignored some characteristics of the offspring such as their sex, despite strong evolutionary and demographic implications of sex ratio variation. We review recent studies that examine associations between glucocorticoids, the main class of stress hormones, and offspring sex. produces males, the extent and direction of sex ratio bias in response to stress vary in reptiles, birds and mammals. We propose proximate and ultimate explanations for most of these

\section{4 trends.}


28 Among vertebrates, sex can be determined by environmental sex determination (ESD, see

Glossary), genetic sex determination (GSD) or the interaction of both. The phylogenetic distribution of GSD and ESD indicates that transitions between these types of sex

31 determination have occurred many times [1]. While mammals and birds have only GSD, sex32 determining mechanisms have high evolutionary plasticity in fish, reptiles and amphibians.

33 Nevertheless, the sensitivity of the undifferentiated gonad to circulating sex hormones is a common feature shared by many vertebrates, including those with GSD [1]. Over the years, the hypothesis that glucocorticoids (GCs), the main class of stress hormones (Box 1), would interact with sex hormones to influence the sexual fate of the gonad has emerged following numerous laboratory studies. In parallel, recent technological advances in stress measurements (Box 1) have allowed the quantification of GCs in free-ranging animals, enabling the discovery of a link between GC levels and sex ratio biases in natural environments. Given GCs act to convert stressful stimuli into physiological signals, they are promising candidates for the translation of environmental, social and state-dependent factors into adaptive sex allocation.

Here we review the body of evidence that GCs influence sex determination and bias the offspring sex ratio across vertebrates (Figure 1). This can happen directly in species with ESD or indirectly through maternal effects. We then explore the potential evolutionary causes explaining why, in a stressful environment, one sex would be more adaptive than the other in

47 animals with either ESD or GSD. 
51 In fish, studies conducted to date have tended to show that at sub-optimal temperatures (i.e.

52 very high or very low), low $\mathrm{pH}$, continuous lighting or high density - each of which can be considered to be a stressful condition [2] - more males are produced (Figure 1). In fish, sex determination can occur at different stages of the life cycle: at fertilization or later in development (egg or larval stage). The undifferentiated gonad of fish is extremely labile and sensitive to external factors [1]. In recent years, the link between stressful environmental conditions and GCs has been established as being a major factor influencing sex determination in fish. For instance, high cortisol levels at the egg stage can override genetic sex in species with GSD such as the medaka (Oryzias latipes) [3] and rainbow trout (Oncorhynchus mykiss) [4]. High temperatures during the larval stage can trigger higher

cortisol production that directly influence the sex of individuals in the Pejerrey (Odontesthes bonariensis) [5] and Japanese flounder (Paralichthys olivaceus) [6]. Other stressful conditions applied at the larval stage such as constant light exposure [7], stressful tank color [8] and high fish density [9] activate the cortisol synthesis pathway and lead to a higher production of males in various fish species (Figure 1). Although this has not been formally tested in natural conditions with controlled quantities of cortisol, administering cortisol to three-spotted wrasse (Halichoeres trimaculatus) induced a female-to-male sex change [10]. In all these examples, more males are produced when stress is applied during the critical period of sex determination or sex differentiation, affecting the primary sex ratio (PSR) (Box 2).

In crocodilians, low temperatures over the egg laying period produce an all-female population, but in lepidosauria (lizards and snakes) and testudines (turtles) temperature sexdetermination is species-specific [11]. When stressed by suboptimal temperatures, corticosterone production rises, even with a relatively small temperature increase, as demonstrated in alligator lizards (Elgaria) [12]. However, the effects of corticosterone exposure on the sex of reptilian offspring has rarely been studied and the few results 
available are not as clear as for fish (Figure 1). Although, investigation of gene expression of the key genes (POMC and $C R H-B P)$ involved in the GC production cascade and regulation showed an association between high temperature, stress and feminization in the Australian central bearded dragon (Pogona vitticeps) [13], another recent study reported a direct link between epigenetic mechanisms and temperature-dependent sex determination in the redeared slider turtle (Trachemys scripta) [14], ruling out GCs as an essential factor, at least in this species. Experimentally introducing corticosterone to developing eggs affected the sex ratio of two lizard species (Amphibolurus muricatus and Bassiana duperreyi) in an opposite pattern [15] (Figure 1), but did not affect sex ratio of the mallee dragon, Ctenophorus fordi [16]. A recent study on the broad-snouted caiman, Caiman latirostris, exposed eggs to a synthetic GC in an attempt to influence the sex ratio of embryos already exposed to high temperature (that produce $100 \%$ males), the authors were unsuccessful at deviating sex-ratio in favour of females [17]. This suggests either that GCs cannot override the temperature effect or that, in caiman, high levels of GCs would produce male-biased clutches anyway as observed in fish (an hypothesis not evaluated by the authors). More studies are needed to understand if temperature can trigger endogenous production of GCs at the period of reptile sex determination.

\section{Influence of maternal glucocorticoids on offspring sex ratio}

Mother-to-offspring GCs transmission is an evolutionarily conserved mechanism found across vertebrate species [18] and is one of the most studied factors in the maternal effect literature $[19,20]$. However, studies on the relationship between maternal GCs and sex ratio in fish are, to date, lacking. Three different studies investigated the link between maternal stress and sex of reptiles' species with different degree of sex-sensitivity to temperature. Two 
studies on the common lizard (Lacerta vivipara) and the eastern fence lizards (Sceloporus

102 undulatus) artificially exposed gravid females to high corticosterone concentration, with no

103 reported sex-ratio bias [21,22]. In Amphibolurus muricatus, a slightly higher percentage of

104 females was detected in clutches of mothers exposed to high corticosterone concentration $105[23]$.

106 In birds, GCs play an important role in sex-ratio biases (Figure 1). Initial studies in 107 captive and free-ranging species showed that elevated levels of maternal GCs are associated 108 with female-biased sex ratios [24-27]. These studies used implants to increase corticosterone 109 levels before nesting and suggested that maternal corticosterone influences PSR. In addition, 110 elevated maternal corticosterone levels can induce higher mortality in male offspring later in 111 development, affecting secondary sex ratio (SSR) [28]. However, recent studies show that 112 the picture is more complex than initially thought: females with elevated corticosterone levels 113 produce more male offspring in Gouldian finches (Erythrura gouldiae), zebra finches 114 (Taeniopygia guttata), chickens (Gallus gallus), and ovenbirds (Seiurus aurocapilla) [29-32].

115 Corticosterone injections to female chickens $5 \mathrm{~h}$ prior to the expected time of ovulation biased 116 sex ratio towards males [31], whereas injections one hour later biased sex ratios towards 117 females [33]. More work is needed to determine whether this unexpected difference is 118 biologically significant. Most avian studies have been performed in controlled conditions and, 119 the three-way association between environmental stressors, maternal corticosterone, and PSR 120 remains to be investigated.

121 In many species of mammals, food deprivation and other environmental perturbations 122 during gestation are typically associated with female-biased sex-ratios at birth [34]. However, 123 results from studies that have measured maternal physiological stress are variable (Figure 1).

124 While some studies have shown a positive relationship between maternal GC levels and 125 female bias in the sex ratio [35-39], others have shown a negative relationship [40-42], or no 
relationship at all [43-48]. Over half of all those studies have been conducted in rodent

127 species (Figure 1). In this group, results are still mixed, but the direction of the pattern, when 128 present, is rather homogeneous: four out of five studies show a decrease in the proportion of 129 male offspring at birth as maternal GCs increased (Figure 1). In humans, a variety of a priori 130 stressful events, ranging from terrorist attacks [49] to death of a close family member [50] 131 were associated with decreases in the proportion of male births, but only two studies

132 measured mother's cortisol with different results (Figure 1). One recent study investigating 133 the link between GC levels of twenty mother howler monkeys (Alouatta pigra) and forest 134 fragmentation [41] showed that females always gave birth to females when their fecal GCs 135 were below a threshold of $200 \mathrm{ng} . \mathrm{g}^{-1}$ around the time of conception $(\mathrm{n}=16)$, while, above 136 this threshold, they consistently produced males $(n=19)$. If future work with a much larger 137 sample size confirms this extraordinary pattern, we should worry about the possible impact of 138 anthropogenic pressures on sex ratios of animals.

\section{Timing of sex ratio and glucocorticoid measurements}

141 The sex ratio can vary in response to stress at several developmental time points. Sex-ratio 142 bias can occur around the time of conception and sexual differentiation (affecting the PSR), or 143 during late stages of development through differential mortality (affecting the SSR) (Box 2).

144 This differential mortality is likely to have higher fitness costs, especially in monotocous 145 species (producing only a single offspring at a time), since it implies loss of offspring after 146 maternal resources have already been invested. Thus, low-cost mechanisms of offspring sex147 ratio adjustment around the time of sex determination and differentiation are more likely to 148 evolve (Box 2).

149 The relationship between stress at the time of sex determination or differentiation and 150 PSR has been mainly studied in fish and birds (Figure 1). In fish, high GCs at this 
151 developmental point led to a PSR biased toward males. Avian meiosis can be influenced by 152 maternal GCs in response to environmental stress, but the timing of the increase in GCs 153 appears critical in determining the sense of the sex-ratio bias [31,33].

154 In most reptiles, the undifferentiated gonads are sensitive to environmental factors 155 during the middle third of the incubation period and it has been reported that maternal steroids 156 in the eggs can decline by more than $90 \%$ before the sex is determined [11,51]. It is thus 157 possible that most GCs from maternal origin would be metabolized before sex differentiation 158 and that the remaining amount (as little as $10 \%$ of the original present) would be insufficient 159 to influence sexual steroid production, explaining why most studies did not report an effect. 160 The two studies that reported an effect experimentally introduced corticosterone into the egg, 161 probably affecting PSR and led to male sex differentiation [15,17]. On the contrary, when 162 more females were produced in Amphibolurus muricatus, the authors proposed that it was 163 through differential mortality of the sexes, suggesting two distinct mechanisms [15]. An 164 experiment evaluating endogenous GCs production at the time of sex determination is now 165 badly needed.

166 In mammals, the relationship between maternal GC levels and PSR remains 167 unexplored since most studies measure the sex ratio at birth (Figure 1). Several studies, 168 however, support the idea that stress occurring around the time of conception plays a key role 169 in sex determination and even sex differentiation (Box 2). Maternal GCs are generally 170 measured around conception in mammals (Figure 1), but this does not provide insight into 171 when and how GCs act. We cannot discard the possibility that the effects of stress on sex ratio 172 may be delayed rather than immediate. Male fetuses are generally more susceptible to food 173 shortages or other forms of environmental harshness during the last stages of gestation [52].

174 At the moment, it is still unclear whether such sex differences in mortality are the product of 175 sexual selection (faster growth and larger energetic requirements in males) or the result of 
adaptive manipulation of the sex ratio by mothers, or a combination of the two. Thus, studies

177 that measure sex ratio at birth can confound two different process: an adaptive effect of maternal stress near conception on offspring sex ratio and passive consequence of maternal stress on sex-specific fetus mortality. Orzark et al. [53] recently showed that 3- to 6-day-old embryos produced by assisted reproduction in the USA were equally likely to be male and

181 female and while natural abortions during the first trimester were biased towards females,

182 second- and third trimester abortions show a strong bias towards males. This suggests that the direction of sex ratio skews in response to maternal stress could change during gestation.

\section{Adaptive significance}

186 Some cases discussed above may be adaptive, meaning that an animal's tendency to develop 187 as a given sex, after experiencing stress directly or indirectly (through mother's influence), is or has been, under positive selection. If stress-related changes in sex ratio are under positive selection, this implies that one sex would have a higher fitness in stressful conditions (Figure

190 2) or that maternal stress modifies the relative costs of producing male and female offspring.

191 Indeed, in the absence of any relationship between maternal stress and the relative fitness of 192 male offspring and female offspring, potential costs of reproduction may favor the production 193 of the less costly sex by stressed females. In practice, demonstrating adaptive significance is 194 not easy because fitness is often difficult to measure, especially in the wild. Furthermore, GCs 195 and maternal GCs may be correlated with other physiological traits that are directly involved 196 in adaptive manipulations of sex ratios [54]. The crossover patterns shown in Figure 2 have 197 not yet been tested in studies investigating direct links between GCs and sex ratio. It is more 198 surprising that potential adaptive explanations are often ignored (e.g. $[33,35])$. We discuss 199 here potential adaptive explanations for most trends observed in Figure 1. 
202 GCs around sex determination or differentiation may provide a 'weather forecast' of the environmental conditions where individuals are likely to live in the future. Related to this idea

204 is the concept of external predictive adaptive response (PAR) [55]. Theoretical work shows 205 that external PAR should only be favored by selection where the association between early206 and later-life environmental conditions is strong [55]. This is most likely to be true for 207 animals with a fast life-history strategy (short lifespan, early maturity, high fecundity and low 208 per capita investment per offspring)[19]. There is increasing evidence that environmental 209 predictability can determine the strength of selection for sex ratio adjustment [56]. For 210 example, greater sex ratio adjustment is seen in ungulates with shorter gestation periods [57] 211 and primates with shorter maturation times [58], possibly because it is easier for parents to 212 predict the amount of energy they would have available for rearing offspring at the time of 213 conception. Fish and birds have, on average, shorter incubation period than reptiles (Figure 2143 , which could explain why GCs play a more important role in the process of sex ratio 215 adjustment in the former than in the latter. While the absolute proportion of studies reporting 216 stress-related biases in the sex ratio may over-estimate the true frequency of GCs effects, 217 since positive results are more likely to be published than negative ones [59], the relative 218 proportion within groups of vertebrates should not be affected. The influence of GCs on the 219 sex ratio appears to be correlated with the length of the prenatal period across the groups of 220 vertebrates (Figures 1 and 3), but this is not always the case within groups. For example, 221 marsupials and rodents have very short gestation periods compared with primates, but the 222 absence of relationship between maternal GCs and sex-ratio has been reported in each group 223 (Figure 1). It is possible that the adaptive potential of maternal stress extends well beyond 224 simply a match between maternal and offspring environment [60]. 
227 Theoretical models developed by Charnov and Bull [61] predicted that selection should favor ESD over GSD if 1) the environment differentially affects the fitness of male versus females, so that the sex produced is the better adapted to a given environment, 2) individual has little control on the environment it will experience, 3) the environment is patchy, so that all sexes are produced. It results that in optimal conditions (i.e. those that allow long term growth and survival) the sex that will have the greatest absolute fitness benefits will be favored and that in harsh conditions, the sex that will be "less costly" to develop will thrive [62]. With fish, developing in stressful conditions would lead to a sex ratio bias towards males. This prediction involves intrinsic sex difference in life-history traits related to reproduction where males generally mature earlier than females and where the cost of producing sperm is much lower than the cost of developing eggs (review of 98 fish species, [63]). In addition, females generally grow bigger than males [64], highlighting that females may require a higher energy input. So, in a low quality patch with limited resources, developing as a male would be adaptive as it would be physiologically less costly and would allow reproducing quicker [65].

241 At least for fish, cortisol would play an essential role as being a physiological transducer 242 providing information of the patch quality since fasting induces cortisol production [66].

\section{Social factors}

245 Social factors like hierarchical rank shape GC levels in many species of all the above 246 described taxa [67-69]. In hermaphroditic fish, such as clownfish species or the bluehead 247 wrasse (Thalassoma bifasciatum), an abrupt change in the established hierarchy triggers sex248 change, and cortisol regulation likely plays a pivotal role in this process $[69,70]$. Social 249 complexity of many species of birds and mammals often leads to multiple selection pressures 250 on sex ratio adjustment, potentially operating in different directions [71]. The Trivers-Willard 
251 hypothesis [72] states that females in good condition, including those of high social rank,

252 should produce more male offspring in species where sexual selection is strong because

253 relatively few high-quality males will monopolize most of the copulations. Consistent with

254 this, a meta-analysis of data for ungulates provided support for the Trivers-Willard hypothesis

255 [57]. Complications arise when daughters 'inherit' their mother's social rank or adult

256 daughters compete with their mothers for local resources, as is the case with some primates

257 and marsupials [45,73,74]. When this occurs, females of high rank can produce more

258 daughters whereas females of low rank can produce more sons especially when competition

259 for resources is high [58]. In primates, a meta-analysis shows that subordinate individuals

260 produce more cortisol than dominants [75], although this negative relationship between social

261 status and GC production might not be necessarily true for all mammalian species [76].

262 Hence, the diversity of associations between maternal social status and offspring sex may

263 contribute to the differences in the sense of the effect of maternal GCs on offspring sex ratio

264 in mammals.

265 There is also variability in the directional patterns of the stress-induced effects on sex

266 ratio of birds, which may partially be explained by the different types of relationships

267 between mate attractiveness and relative fitness of sons and daughters. In birds, more than

$26890 \%$ of all species are socially monogamous. Mate choice is constrained by the availability of

269 unpaired individuals in the population and having an unattractive mate can be stressful for the

270 female [77-79]. The mate attractiveness hypothesis predicts that females mated to

271 unattractive males should produce more daughters because these daughters will not inherit

272 their father's unelaborated sexual ornaments [80]. However, the reverse prediction exists in

273 Gouldian finches and maybe other, similar species because of the higher mortality for

274 daughters than for sons of genetically incompatible pairs [81]. In both Gouldian and zebra

275 finches, females with elevated corticosterone levels produce more male offspring $[29,30]$. It is 
assumed that such manipulation is under female control, because female birds are the

277 heterogametic sex (Box 2). In species in which males are the heterogametic sex, fathers may 278 play a role in controlling the sex of offspring. Recent advances in mammals shows that the 279 proportion of $\mathrm{X}$ - and $\mathrm{Y}$-chromosome bearing sperm can vary between individuals, reinforcing 280 the need to consider paternal characteristics, including stress, in future studies $([82,83]$, see 281 Outstanding questions).

\section{Predation and sex-biased dispersal}

284 Sex-ratio adjustments in response to stress might be especially important in situations where 285 predation is the main stressor since it is a major evolutionary force acting on prey and changes 286 in predation risk tend to occur slowly rather than varying greatly from one year to the next 287 [84]. Individuals within the same population can be exposed to varying degrees of risk. For instance, young are generally more vulnerable to predation, but their probability of being predated upon sometimes depends on body condition [85]. For individuals subjected to high predation risk, escaping through a higher dispersal potential might constitute an optimal strategy to increase the probability of survival. In this sense, offspring common lizards

292 (Zootoca vivipara) whose mothers were exposed to predator cues disperse three times further 293 than those of non-exposed mothers [86]. Alternatively, one could argue that philopatry would 294 instead increase in offspring of stressed females, as demonstrated in common lizards 295 artificially exposed to GCs [87]. In either case, it is possible that the sex with the highest 296 probability of survival (by staying or migrating more than the other sex) in a dangerous 297 environment would be overproduced. In lizards and fish, there is no clear general pattern of 298 sex-biased dispersal, although juvenile males have been reported to disperse more in some 299 lizards [88] and migrating fish (e.g. Oncorhynchus mykiss [89]). The picture is clearer for 300 mammals in which males generally (but not always) disperse more than females, while the 
reverse is typically observed in birds [90,91]. Hence, if we consider that high dispersal potential is somehow related to differential survival between sexes under high predation, then more males should be produced in the majority of mammals, while more females should be produced in most birds. Studies combining measurements of predation risk, sex ratio and GC levels are now needed to test these predictions.

\section{Concluding Remarks}

The sensitivity of the sex ratio to GC levels appears very variable among vertebrates. The effects are more prevalent in fish and birds than in reptiles and mammals. The different mechanisms of sex determination (genetic vs. environmental) are not sufficient to explain

311 these results because GSD is found in birds and ESD occurs in many species of reptiles.

312 Stress-related sex ratio biases are not necessarily more extreme in fish, but the direction of the sex-ratio skew is uniform in comparison with the other taxa. While it is possible to propose an adaptive explanation for most of these trends, more studies are necessary to test them in a comprehensive meta-analysis. Fitness functions are essential for understanding the adaptive

316 significance of stress-related sex-ratio bias and there are obvious benefits to conducting such 317 investigations in the wild. A better knowledge of the physiological mechanisms that link 318 maternal and environmental stressors to offspring, by taking advantage of both new non319 invasive tools (Box 1) and useful animal models, would also be particularly helpful for an 320 integrative understanding of stress-related sex ratio variation (see Outstanding questions).

322 Acknowledgments:

324 We would like to thank Claus Wedekind, Jean-François Lemaître, Ben Parrott, Kristen 325 Navarra, Michael Sheriff, Andrea Stephens and two anonymous reviewers for constructive 326 comments. We also thank Starrlight Augustine, Bastien Sadoul for their help in collecting 327 Dynamic Energy Budget (DEB) data and Pierre Lopez for drawing species. Finally we thank 
Tony Tebby for English correction and suggestions. B.G. is supported by the European Maritime and Fisheries Fund - 3S (Seabass Sex and Stress) n4320175237.

1 Capel, B. (2017) Vertebrate sex determination: evolutionary plasticity of a fundamental switch. Nat. Rev. Genet. 18, 675-689

2 Schreck, C.B. et al. (2016) Biology of Stress in Fish, Academic Press.

3 Hayashi, Y. et al. (2010) High temperature causes masculinization of genetically female medaka by elevation of cortisol. Mol. Reprod. Dev. 77, 679-686

4 van den Hurk, R. and van Oordt, P.G.W.J. (1985) Effects of natural androgens and corticosteroids on gonad differentiation in the rainbow trout, Salmo gairdneri. Gen. Comp. Endocrinol. 57, 216-222

5 Hattori, R.S. et al. (2009) Cortisol-Induced Masculinization: Does Thermal Stress Affect Gonadal Fate in Pejerrey, a Teleost Fish with Temperature-Dependent Sex Determination? PLoS ONE 4, e6548

6 Yamaguchi, T. et al. (2010) Cortisol Is Involved in Temperature-Dependent Sex Determination in the Japanese Flounder. Endocrinology 151, 3900-3908

3487 Corona-Herrera, G.A. et al. Experimental evidence of masculinization by continuous illumination in a temperature sex determination teleost (Atherinopsidae) model: is oxidative stress involved? J. Fish Biol.DOI: 10.1111/jfb.13651

8 Mankiewicz, J.L. et al. (2013) Masculinizing Effect of Background Color and Cortisol in a Flatfish with Environmental Sex-Determination. Integr. Comp. Biol. 53, 755-765

9 Ribas, L. et al. (2017) Appropriate rearing density in domesticated zebrafish to avoid masculinization: links with the stress response. J. Exp. Biol. 220, 1056-1064

10 Nozu, R. and Nakamura, M. (2015) Cortisol Administration Induces Sex Change from Ovary to Testis in the Protogynous Wrasse, Halichoeres trimaculatus; Sex. Dev. 9, 118124

11 Miyagawa, S. et al. (2018) Environmental Control of Sex Determination and Differentiation in Reptiles. In Reproductive and Developmental Strategies pp. 367-390, Springer, Tokyo

12 Telemeco, R.S. and Addis, E.A. (2014) Temperature has species-specific effects on corticosterone in alligator lizards. Gen. Comp. Endocrinol. 206, 184-192

13 Deveson, I.W. et al. (2017) Differential intron retention in Jumonji chromatin modifier genes is implicated in reptile temperature-dependent sex determination. Sci. Adv. 3, e1700731

14 Ge, C. et al. (2018) The histone demethylase KDM6B regulates temperature-dependent sex determination in a turtle species. Science 360, 645-648

15 Warner, D.A. et al. (2009) Corticosterone Exposure during Embryonic Development Affects Offspring Growth and Sex Ratios in Opposing Directions in Two Lizard Species with Environmental Sex Determination. Physiol. Biochem. Zool. 82, 363-371

16 Uller, T. et al. (2009) Sex-specific developmental plasticity in response to yolk corticosterone in an oviparous lizard. J. Exp. Biol. 212, 1087-1091

17 Iungman, J.L. et al. (2015) Are Stress-Related Hormones Involved in the TemperatureDependent Sex Determination of the Broad-Snouted Caiman? South Am. J. Herpetol. 10, 41-49 
18 Thayer, Z.M. et al. (2018) Impact of prenatal stress on offspring glucocorticoid levels: A phylogenetic meta-analysis across 14 vertebrate species. Sci. Rep. 8, 4942

19 Sheriff, M.J. and Love, O.P. (2013) Determining the adaptive potential of maternal stress. Ecol. Lett. 16, 271-280

20 Mousseau, T.A. and Fox, C.W. (1998) The adaptive significance of maternal effects. Trends Ecol. Evol. 13, 403-407

21 Owen, D. a. S. et al. Sex-dependent effects of maternal stress: Stressed moms invest less in sons than daughters. J. Exp. Zool. Part Ecol. Integr. Physiol. 0,

22 Uller, T. et al. (2005) Is sexual dimorphism affected by the combined action of prenatal stress and sex ratio? J. Exp. Zoolog. A Comp. Exp. Biol. 303, 1110-1114

23 Warner, D.A. et al. (2007) Maternal nutrition affects reproductive output and sex allocation in a lizard with environmental sex determination. Proc. R. Soc. Lond. B Biol. Sci. 274, 883-890

24 Pike, T.W. and Petrie, M. (2005) Maternal body condition and plasma hormones affect offspring sex ratio in peafowl. Anim. Behav. 70, 745-751

25 Pike, T.W. and Petrie, M. (2006) Experimental evidence that corticosterone affects offspring sex ratios in quail. Proc. R. Soc. B Biol. Sci. 273, 1093-1098

26 Bonier, F. et al. (2007) Maternal corticosteroids influence primary offspring sex ratio in a free-ranging passerine bird. Behav. Ecol. 18, 1045-1050

27 Goerlich-Jansson, V.C. et al. (2013) Manipulation of primary sex ratio in birds: Lessons from the Homing Pigeon (Columba livia domestica). Integr. Comp. Biol. 53, 902-912

28 Love, O.P. et al. (2005) Stress Hormones: A Link between Maternal Condition and SexBiased Reproductive Investment. Am. Nat. 166, 751-766

29 Pryke, S.R. et al. (2011) Maternal stress to partner quality is linked to adaptive offspring sex ratio adjustment. Behav. Ecol. 22, 717-722

30 Gam, A.E. et al. (2011) Acute corticosterone treatment prior to ovulation biases offspring sex ratios towards males in zebra finches Taeniopygia guttata. J. Avian Biol. 42, 253-258

31 Pinson, S.E. et al. (2011) Acute Corticosterone Administration during Meiotic Segregation Stimulates Females to Produce More Male Offspring. Physiol. Biochem. Zool. 84, 292-298

32 Leshyk, R. et al. (2012) Logging affects fledgling sex ratios and baseline corticosterone in a forest songbird. PLOS ONE 7, 1-7

33 Pinson, S.E. et al. (2015) Timing matters: corticosterone injections 4 h before ovulation bias sex ratios towards females in chickens. J. Comp. Physiol. [B] 185, 539-546

34 Navara, K.J. (2018) Choosing Sexes: Mechanisms and Adaptive Patterns of Sex Allocation in Vertebrates, Springer International Publishing.

35 Chason, R.J. et al. (2012) Preconception stress and the secondary sex ratio: A prospective cohort study. Fertil. Steril. 98, 937-941

36 Geiringer Erich (1961) Effect of ACTH on sex ratio of the albino rat. Proc. Soc. Exp. Biol. Med. 106, 752-754

37 Lane, E.A. and Hyde, T.S. (1973) Effect of maternal stress on fertility and sex ratio: A pilot study with rats. J. Abnorm. Psychol. 82, 78-80

38 Pratt, N.C. and Lisk, R.D. (1990) Dexamethasone can prevent stress-related litter deficits in the golden hamster. Behav. Neural Biol. 54, 1-12

39 Mahmoodkhani, M. et al. (2018) Pre-pregnancy stress suppressed the reproductive systems in parents and changed sex ratio in offspring. J. Appl. Biomed. 16, 370-377

40 Moore, E.P.B. et al. (2015) High density, maternal condition, and stress are associated with male-biased sex allocation in a marsupial. J. Mammal. 96, 1203-1213

41 Rangel-Negrín, A. et al. (2017) Maternal glucocorticoid levels affect sex allocation in 
black howler monkeys. J. Zool. DOI: 10.1111/jzo.12503

42 Ryan, C.P. et al. (2012) Stress-induced sex ratios in ground squirrels: support for a mechanistic hypothesis. Behav. Ecol. 23, 160-167

43 Bae, J. et al. (2017) Preconception stress and the secondary sex ratio in a populationbased preconception cohort. Fertil. Steril. 107, 714-722

44 Monclús, R. et al. (2011) Older mothers follow conservative strategies under predator pressure: The adaptive role of maternal glucocorticoids in yellow-bellied marmots. Horm. Behav. 60, 660-665

45 Schwanz, L.E. and Robert, K.A. (2014) Proximate and ultimate explanations of mammalian sex allocation in a marsupial model. Behav. Ecol. Sociobiol. 68, 1085-1096

46 Helle, S. et al. (2008) Female field voles with high testosterone and glucose levels produce male-biased litters. Anim. Behav. 75, 1031-1039

47 Pollard, I. (1984) Effects of stress administered during pregnancy on reproductive capacity and subsequent development of the offspring of rats: Prolonged effects on the litters of a second pregnancy. J. Endocrinol. 100, 301-306

48 Mendl, M. et al. (1995) Maternal social status and birth sex ratio in domestic pigs: an analysis of mechanisms. Anim. Behav. 50, 1361-1370

49 Bruckner, T.A. et al. (2010) Male fetal loss in the U.S. following the terrorist attacks of September 11, 2001. BMC Public Health 10, 273

50 Hansen, D. et al. (1999) Severe periconceptional life events and the sex ratio in offspring: follow up study based on five national registers. Br. Med. J. 319, 548-549

51 Lance, V.A. Is regulation of aromatase expression in reptiles the key to understanding temperature-dependent sex determination? J. Exp. Zool. Part Ecol. Genet. Physiol. 311A, 314-322

52 Clutton-Brock, T.H. (1991) The evolution of parental care, Princeton University Press.

53 Orzack, S.H. et al. (2015) The human sex ratio from conception to birth. Proc. Natl. Acad. Sci. 112, E2102-E2111

54 Douhard, M. (2017) Offspring sex ratio in mammals and the Trivers-Willard hypothesis: In pursuit of unambiguous evidence. BioEssays 39, 1-10

55 Nettle, D. et al. (2013) The evolution of predictive adaptive responses in human life history. Proc. R. Soc. B Biol. Sci. 280, 20131343

56 West, S.A. (2009) Sex allocation, Princeton University Press.

57 Sheldon, B.C. and West, S.A. (2004) Maternal dominance, maternal condition, and offspring sex ratio in ungulate mammals. Am. Nat. 163, 40-54

58 Schino, G. (2004) Birth sex ratio and social rank: Consistency and variability within and between primate groups. Behav. Ecol. 15, 850-856

59 Rosenthal, R. (1979) The file drawer problem and tolerance for null results. Psychol. Bull. 86, 638-641

60 Sheriff, M.J. et al. (2018) Error management theory and the adaptive significance of transgenerational maternal-stress effects on offspring phenotype. Ecol. Evol. 8, 64736482

61 Charnov, E.L. and Bull, J. (1977) When is sex environmentally determined? Nature 266, 828-830

62 Frank, S.A. and Swingland, I.R. (1988) Sex ratio under conditional sex expression. J. Theor. Biol. 135, 415-418

63 Hayward, A. and Gillooly, J.F. (2011) The Cost of Sex: Quantifying Energetic Investment in Gamete Production by Males and Females. PLoS ONE 6, e16557

64 Pauly, D. (2019) Female Fish Grow Bigger - Let's Deal with It. Trends Ecol. Evol. DOI: 10.1016/j.tree.2018.12.007

65 Geffroy, B. and Bardonnet, A. (2016) Sex differentiation and sex determination in eels: 
consequences for management. Fish Fish. 17, 375-398

66 Barcellos, L.J.G. et al. (2010) The effects of fasting on cortisol, blood glucose and liver and muscle glycogen in adult jundiá Rhamdia quelen. Aquaculture 300, 231-236

67 Creel, S. et al. (2013) The ecology of stress: Effects of the social environment. Funct. Ecol. 27, 66-80

68 Greenberg, N. et al. (1984) Social status, gonadal state, and the adrenal stress response in the lizard, Anolis carolinensis. Horm. Behav. 18, 1-11

69 Olivotto, I. and Geffroy, B. (2017) Clownfish. In Marine Ornamental Species Aquaculture (Calado, R. et al., eds), pp. 177-199, John Wiley \& Sons, Ltd

70 Goikoetxea, A. et al. (2017) Stress and sex: does cortisol mediate sex change in fish? Reproduction 154, R149-R160

71 Cockburn, A. et al. (2002) Sex ratios in birds and mammals: can the hypotheses be disentangled. Sex Ratios Concepts Res. Methods DOI: http://dx.doi.org/10.1017/CBO9780511542053

72 Trivers, R.L. and Willard, D.E. (1973) Natural selection of parental ability to vary the sex ratio of offspring. Science 173, 90-92

73 Silk, J.B. (1983) Local resource competition and facultative adjustment of sex ratios in relation to competitive abilities. Am. Nat. 121, 56-66

74 Simpson, M.J.A. and Simpson, A.E. Birth sex ratios and social rank in rhesus monkey mothers. , Nature, 300. (1982) , 440-441

75 Abbott, D.H. et al. (2003) Are subordinates always stressed? A comparative analysis of rank differences in cortisol levels among primates. Horm. Behav. 43, 67-82

76 Creel, S. (2001) Social domiance and stress hormones. Trends Ecol. Evol. 16, 491-497

77 Griffith, S.C. et al. (2011) Constrained mate choice in social monogamy and the stress of having an unattractive partner. Proc. R. Soc. B Biol. Sci. 278, 2798-2805

78 Pike, T.W. and Petrie, M. (2005) Offspring sex ratio is related to paternal train elaboration and yolk corticosterone in peafowl. Biol. Lett. 1, 204-207

79 Mougeot, F. et al. (2016) Parasites, mate attractiveness and female feather corticosterone levels in a socially monogamous bird. Behav. Ecol. Sociobiol. 70, 277-283

80 Booksmythe, I. et al. (2017) Facultative adjustment of the offspring sex ratio and male attractiveness: a systematic review and meta-analysis. Biol. Rev. 92, 108-134

81 Pryke, S.R. and Griffith, S.C. (2009) Genetic incompatibility drives sex allocation and maternal investment in a polymorphic finch. Science 323, 1605-1607

82 Edwards, A.M. and Cameron, E.Z. (2014) Forgotten fathers: Paternal influences on mammalian sex allocation. Trends Ecol. Evol. 29, 158-164

83 Douhard, M. (2018) The role of fathers in mammalian sex allocation. Mammal Rev. 48, 67-74

84 Boonstra, R. (2013) Reality as the leading cause of stress: rethinking the impact of chronic stress in nature. Funct. Ecol. 27, 11-23

85 Ronget, V. et al. (2017) The 'Evo-Demo' Implications of Condition-Dependent Mortality. Trends Ecol. Evol. 32, 909-921

86 Bestion, E. et al. (2014) Maternal exposure to predator scents: offspring phenotypic adjustment and dispersal. Proc. R. Soc. Lond. B Biol. Sci. 281, 20140701

87 Fraipont, M.D. et al. Increased pre-natal maternal corticosterone promotes philopatry of offspring in common lizards Lacerta vivipara. J. Anim. Ecol. 69, 404-413

88 Liebgold, E.B. et al. (2011) Female philopatry and male-biased dispersal in a directdeveloping salamander, Plethodon cinereus. Mol. Ecol. 20, 249-257

89 Brunelli, J.P. et al. (2010) Deep divergence and apparent sex-biased dispersal revealed by a Y-linked marker in rainbow trout. Mol. Phylogenet. Evol. 56, 983-990

90 Greenwood, P.J. (1980) Mating systems, philopatry and dispersal in birds and mammals. 
Anim. Behav. 28, 1140-1162

91 Trochet, A. et al. (2016) Evolution of Sex-Biased Dispersal. Q. Rev. Biol. 91, 297-320

92 Romero, L.M. (2004) Physiological stress in ecology: lessons from biomedical research. Trends Ecol. Evol. 19, 249-255

93 Bonnot, N.C. et al. (2018) Who's afraid of the big bad wolf? Variation in the stress response among personalities and populations in a large wild herbivore. Oecologia DOI: 10.1007/s00442-018-4174-7

94 Dettmer, A.M. et al. (2014) Population density-dependent hair cortisol concentrations in rhesus monkeys (Macaca mulatta). Psychoneuroendocrinology 42, 59-67

95 Messina, S. et al. (2018) Physiological and immunological responses of birds and mammals to forest degradation: A meta-analysis. Biol. Conserv. 224, 223-229

96 Wessling, E.G. et al. (2018) The costs of living at the edge: Seasonal stress in wild savanna-dwelling chimpanzees. J. Hum. Evol. DOI: 10.1016/j.jhevol.2018.03.001

97 Dantzer, B. et al. (2017) Social conflict and costs of cooperation in meerkats are reflected in measures of stress hormones. Behav. Ecol. 28, 1131-1141

98 Geffroy, B. et al. (2015) How Nature-Based Tourism Might Increase Prey Vulnerability to Predators. Trends Ecol. Evol.

99 Mommsen, T.P. et al. (1999) Cortisol in teleosts: dynamics, mechanisms of action, and metabolic regulation. Rev. Fish Biol. Fish. 9, 211-268

100 Hayward, L.S. and Wingfield, J.C. (2004) Maternal corticosterone is transferred to avian yolk and may alter offspring growth and adult phenotype. Gen. Comp. Endocrinol. 135, 365-371

101 Dufty, A.M. et al. (2002) Hormones, developmental plasticity and adaptation. Trends Ecol. Evol. 17, 190-196

102 Dettmer, A.M. et al. (2018) Cortisol in Neonatal Mother's Milk Predicts Later Infant Social and Cognitive Functioning in Rhesus Monkeys. Child Dev. 89, 525-538

103 Dickens, M.J. and Romero, L.M. (2013) A consensus endocrine profile for chronically stressed wild animals does not exist. Gen. Comp. Endocrinol. 191, 177-189

104 Dantzer, B. et al. (2014) Measures of physiological stress : a transparent or opaque window into the status, management and conservation of species ? Physiology 2, 1-18

105 Sheriff, M.J. et al. (2011) Measuring stress in wildlife: Techniques for quantifying glucocorticoids. Oecologia 166, 869-887

106 Palme, R. (2019) Non-invasive measurement of glucocorticoids: Advances and problems. Physiol. Behav. 199, 229-243

107 Sadoul, B. and Geffroy, B. Measuring cortisol, the major stress hormone in fishes. $J$. Fish Biol. DOI: 10.1111/jfb.13904

108 Ideta, A. et al. (2009) Subjecting holstein heifers to stress during the follicular phase following superovulatory treatment may increase the female sex ratio of embryos. $J$. Reprod. Dev. 55, 529-533

109 Guiguen, Y. et al. (2010) Ovarian aromatase and estrogens: A pivotal role for gonadal sex differentiation and sex change in fish. Gen. Comp. Endocrinol. 165, 352-366

110 Panza, S. et al. (2016) Glucocorticoid Receptor as a Potential Target to Decrease Aromatase Expression and Inhibit Leydig Tumor Growth. Am. J. Pathol. 186, 13281339

111 Goto, M. et al. (2006) In humans, early cortisol biosynthesis provides a mechanism to safeguard female sexual development. J. Clin. Invest. 116, 953-960

112 El-Maouche, D. et al. (2017) Congenital adrenal hyperplasia. Lancet Lond. Engl. 390, 2194-2210

\section{Box 1: Stress and glucocorticoids}


577 A widely used definition of "stress" is "the physiological cascade of events that occurs when 578 the organism is attempting to resist death or reestablish homeostatic norms in the face of 579 insult" [2]. The secretion and synthesis of glucocorticoids (GCs) is considered the primary 580 endocrine response (together with the release of catecholamines) to unpredictable or 581 uncontrollable stimuli (stressors) in the environment [92]. In the wild, high GC levels could 582 result from many different types of stressors including predator attack or presence [93], high 583 population density [94], habitat degradation [95], food shortages [96], social conflict [97] or 584 human activities [98]. Here we considered all GCs increase resulting from these stressful 585 cases, although it is worth noting that harsh conditions do not necessarily provoke a stress 586 response [76].

587 Stressors can be classified as acute or chronic, with acute being of short duration (minutes to 588 hours) and chronic being days to months [84]. Some acute stressors can, however, have long589 lasting consequences. The effects of stress may even span generations. These 590 transgenerational effects are often due to the transfer of GCs from the mother to the progeny, 591 through eggs in fish [99], birds [100] and reptiles [101] or blood and milk in mammals [102].

592 The reliability of GCs to assess chronic stress has recently been questioned [103]. Indeed, 593 animals can show unchanged or lower GC levels after exposure to chronic stress [103]. Some 594 of this ambiguity may be due techniques used to quantify GC levels and habituation to the 595 chronic stress methodology in the laboratory studies [104]. Although blood sampling has been 596 commonly used for measuring GC concentrations, it is not necessary the most pertinent 597 method [105]. Integrated and non-invasive measure of GCs, such as those from feces, hair, 598 scales, feathers, surrounding water, may more reflect the cumulative exposure of individuals 599 to GCs rather than repeated measurements of GCs in blood or saliva [104-107]. The 600 evaluation of several stress response parameters remains strongly recommended for a correct 
interpretation of data [106].

602

603

604

605

606

607

608

609

610

611

612

613

614 mechanism involves control of aromatase (enzyme converting androgens into estrogens). In

621 fish, as in reptiles, aromatase is central to determining the sexual fate of ambisexual gonads 622 [51,109]. This enzyme could be controlled by GCs, as demonstrated in the Japanese flounder 623 where complex cortisol/glucocorticoid receptors (GR) bind to the glucocorticoid response 624 overproduction of males [6] (Figure element (GRE) on the aromatase promoter to decrease aromatase expression, resulting in an

$626 \mathrm{GCs} / \mathrm{GR} /$ aromatase appears to be well conserved in human testicular Leydig cell tumors, also 627 triggering a decrease in estrogen production [110]. In a normal functioning, the appropriate 
628 intrauterine sexual steroid balance is also fundamental in humans, since female differentiation

629 (occurring from 7 to 12 weeks post conception) is vulnerable to androgen before the 630 protective placental aromatase appears [111]. During this sex differentiation period, standard

631 cortisol production reduces androgen synthesis, allowing normal female sex differentiation

632 [111]. Remarkably, in humans, mutations in the cortisol synthesis pathway, decreasing 633 cortisol production, is involved in the virilization of 46, XX girls with congenital adrenal 634 hyperplasia [112]. Cortisol thus appears to plays a key role in different species concerning the 635 balance of sexual steroids. The extent to which stress-linked cortisol production would affect 636 human and other animals sex differentiation, remains an open question. We nevertheless 637 recommend systematically investigating the genotypic sex of focal species to detect for 638 potential stress-induced sex reversal.

639

640 Figure I An overview of mechanisms by which glucocorticoids (GCs) can influence sex 641 determination and sex differentiation in vertebrates. X-CBS: X-chromosome-bearing sperm; 642 Y-CBS: Y-chromosome-bearing sperm. 
Glossary

646 Acute stressor: short-term environmental challenges to the physiology of the animal.

647 Chronic stressor: long-term environmental challenges to the physiology of the animal.

648 Cortisol: The main glucocorticoid hormone produced by fish and most mammals through the 649 hypothalamic-pituitary-adrenocortical (Interrenal for fish) axis, and released in response to 650 stressors.

651 Corticosterone: The main glucocorticoid hormone produced by birds, reptiles and 652 amphibians and released in response to stressors.

653 Environmental sex determination (ESD): sex is determined by external factors, such as 654 temperature, water ( $\mathrm{pH}$, oxygen), or density of conspecifics.

655 External predictive adaptive response (PAR): a form of developmental plasticity in 656 response to environmental cues acting early in life, but where the advantages of the induced 657 phenotype manifest only later in life.

658 Genetic sex determination (GSD): sex is determined by the presence or absence of genes, 659 generally located on sex chromosomes.

660 Glucocorticoids (GCs): a class of steroid hormones present in all vertebrates, involved in 661 many physiological processes such as response to stress.

662 Heterogametic sex: the sex that has two different sex chromosomes (male XY in mammals; 663 female ZW in birds).

664 Homogametic sex: the sex that has two identical sex chromosomes (female XX in mammals; 665 male ZZ in birds).

666 Primary sex ratio (PSR): initial sex ratio when sexual differentiation occurs.

667 Offspring sex ratio: proportion of offspring that are males at different scopes (species, 668 population, individual, clutch/litter). Here, sex-ratio is generally considered at the clutch/litter 669 level. 
670 Secondary sex ratio (SSR): sex ratio after sex differentiation that results from potential sex-

671 specific mortality before birth.

672 Sex determination: any of the various mechanisms in which the sex of the individual animal

673 is determined.

674 Sex differentiation: The process following sex determination, where the development of a

675 undifferentiated primordial gonad into testes or ovaries occurs.

Figure Caption.

679 Figure 1. Effects of glucocorticoids (GCs) on the sex ratio of different species of fish, reptiles, 680 birds and mammals (animal design: Pierre Lopez). Glucocorticoids were applied or measured: 681 before conception; around sex determination or differentiation; during the late phase of gestation or development. Highs levels of stress can induce increases in the proportion of males (animals in blue), reductions in the proportion of males (animals in red) or no change at all (animals in grey). GCs were measured in the mother for all mammals and birds (except 34), while for fish and most reptiles (except 23; 24) it was measured on the animal itself. Number in bracket indicates the corresponding reference. Full black circles indicate species for which primary sex ratio was assessed, while empty circles indicate species for which secondary sex ratio was determined. Note that for the two marsupial species $(42,47)$ the exact timing of GCs measurement relative to pouch status is unknown due to its particular development.* Caimans were exposed to a temperature protocol producing $100 \%$ males and exposure to GC did not change this pattern.

693 Figure 2. The pattern of fitness that can be observed if the sex-ratio adjustments in response to 694 stress are adaptive. The three panels represent adaptive scenarios because the lines cross. Sex

695 A can be either male or female. The sex that gains the greater fitness benefits in a hazardous environment (sex A) should be preferred under high stress conditions, and the other sex (sex B) should be preferred under low stress conditions.

699 Figure 3 Duration of incubation or gestation time (days) as a potential driver of sex-ratio adjustments in response to stress in teleost fish $(n=53)$, birds $(n=447)$, reptiles $(n=7)$ and 
mammals $(n=420)$. Box plots show the mean represented by black diamond, the median

702 represented by lines, first and third quartiles as well as outliers represented by full circles

703

704 environment around sex determination and the offspring's future environment. sex determination processes of various species.

- A combination of field and laboratory studies is required to understand the extent to

724 which stress influences offspring sex from conception to birth. outside the box. Note that 14 outliers > 400 days were omited in mammals for design purpose. Signs and background color represent the strength of the match between the environment experienced at the time of sex determination (SD) and the one experienced by newly born offspring, with "+" indicating a strong match and "-." indicating a weak match. The dashed line represents the average SD for most represented species (note that it could occur during incubation or later for some fish and reptiles). Data were extracted from the dynamic energetic budget website (https://www.bio.vu.nl/thb/deb/) reporting the life history traits of more than 1500 species.

Highlights

- Offspring sex ratios have been shown to correlate with environmental stressors and maternal stress in many vertebrate species.

- $\quad$ There is an adaptive advantage for parents to produce the sex that is more likely to survive and reproduce in a future hazardous environment.

- In the fastest life histories, there is more likely to be a close good match between the - Glucocorticoids are key messengers of environmental contexts that likely influence the which stress influences offspring sex from conception to birth.

\section{OUTSTANDING QUESTIONS:}

Although we have demonstrated that there is a relationship between stress and sex ratio and 
that this varies strongly between vertebrates, many areas are still little understood. Questions

730 for the future include:

731 - Do chronic and acute increases in GCs act on sex determination through similar $732 \quad$ mechanisms?

733 - Do increases in GCs during the sex differentiation period in reptiles change the sex $734 \quad$ ratio?

735 - To what extent could sexual dimorphism in other life history traits such as age at 736 maturation, growth or metabolism drive the production of a certain sex under stressful conditions?

- To what extent key life history traits, allowing to distinct fast and slow selected species, explain the sensitivity of offspring's sex to stress?

- Does paternal stress explain sex ratio skews that are not explained by maternal stress in species, such as mammals; where males are the heterogametic sex?

- Are sex ratio skews the product of differences in the susceptibility of the two sexes to environmental stressors or are they the consequence of adaptive adjustment by parents?

- Does the presence of the chorion, which potentially protects the embryo during initial phase of sex differentiation mechanisms from external stressors, increase selection for a master sex determining genes in some fish species?

- Is the differential mortality between sexes linked with stress at the earliest stages of development?

- To what extent are epigenetic mechanisms and stress-related information linked?

- Do GCs act alone to skew sex ratios or in combination with other non-sexual steroid molecules such as glucose? 


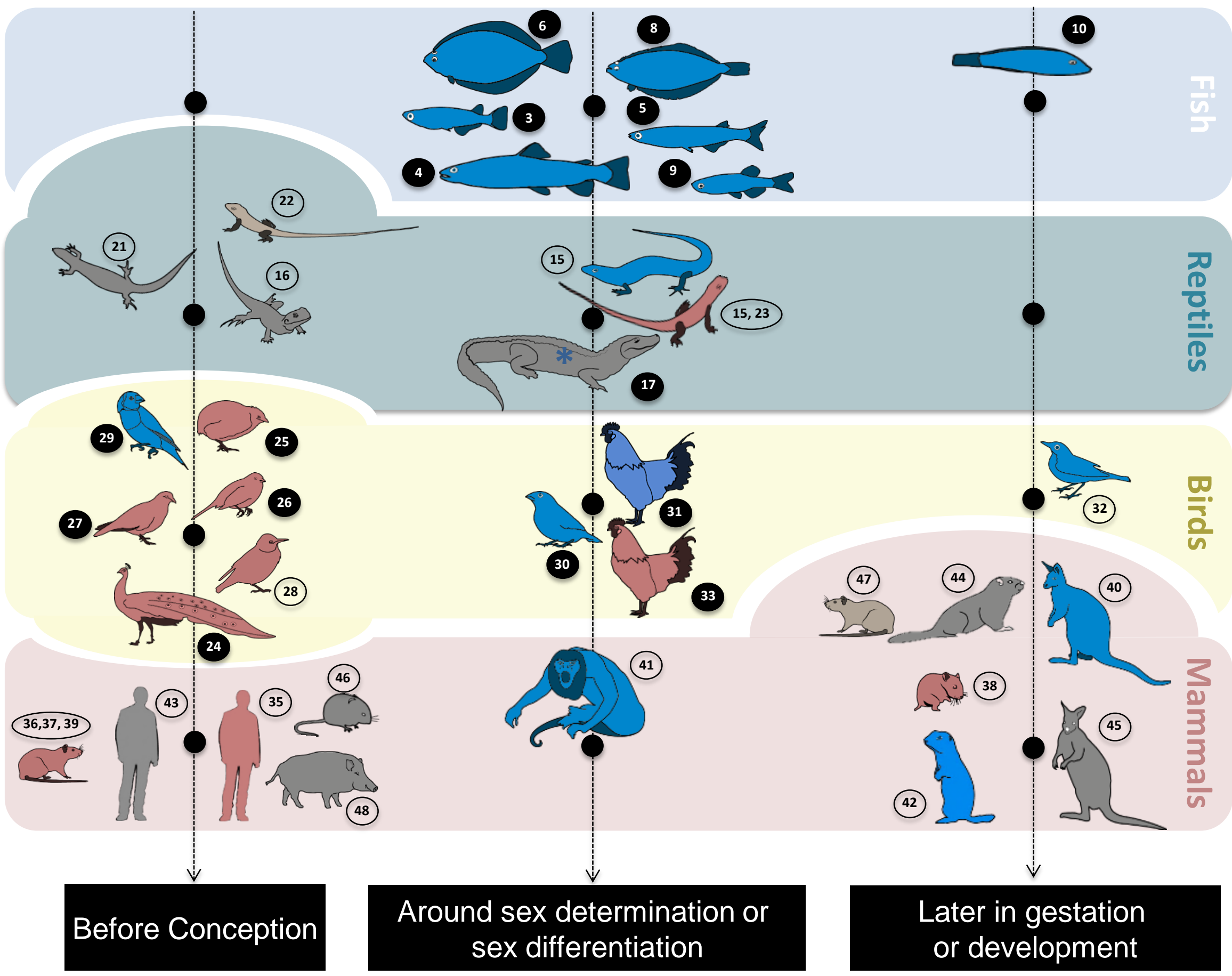




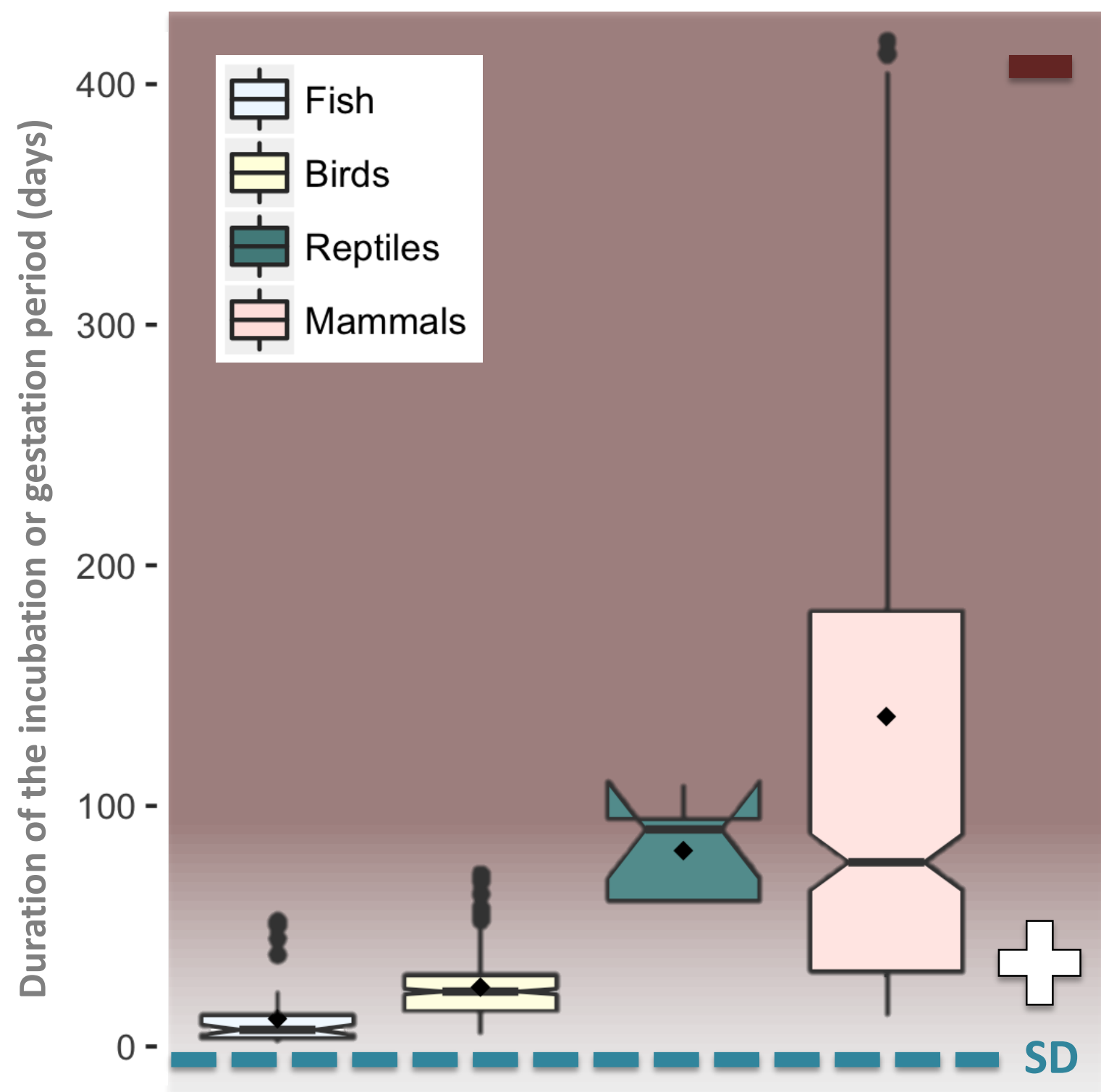

Stressful environment 